Original Article

\title{
LOCALIZATION AND ORIENTATION OF METHOXY FLAVONOIDS IN DPPC BILAYERS: EFFECT ON THEIR ANTI-PROLIFERATIVE ACTIVITY
}

\author{
RAGINI SINHA ${ }^{\mathrm{a}^{*}}$, URMILA J. JOSHI ${ }^{\mathrm{b}}$, AKSHADA JOSHI ${ }^{\mathrm{b}}$, GIRJESH GOVIL \\ aNational Facility for High Field NMR, Tata Institute of Fundamental Research, Homi Bhabha Road, Mumbai 400005, India, bPrincipal K M \\ Kundnani College of Pharmacy, Cuffe Parade, Mumbai 400005, India \\ Email: raginisinha1@rediffmail.com \\ Received: 24 Dec 2017, Revised and Accepted: 05 Feb 2018
}

\begin{abstract}
Objective: Flavones and flavonols are an important class of naturally occurring flavonoids. They are well known for their pharmacological activity. This activity is associated with the ability of flavones and flavonols to influence membrane-dependent processes. In this paper, we have reported localization, orientation and interaction, of four synthesized flavone/flavonols with 1, 2-dipalmitoyl-sn-glycero-3-phosphocholine (DPPC) bilayers. These are compared with standard flavone; chrysin (CHY) and flavonol Quercetin (QUE).
\end{abstract}

Methods: The molecules studied are 4'-methoxy flavone (MF), 3',4'-dimethoxyflavone (DMF), 4'-methoxyflavonol (MF-ol) and 3',4'dimethoxyflavonol (DMF-ol). The techniques used are Differential Scanning Calorimetry (DSC) and multi-nuclear NMR.

Results: Highest binding to lipid bilayers is shown by DMF, followed by QUE. Based on DSC studies it is seen, that maximum interaction of MF and DMF, takes place with the hydrophobic core of lipid bilayers. DMF-ol shows formation of a heterogeneous system at higher concentrations. The ${ }^{1} \mathrm{H}$ NMR spectra of unilamellar vesicles of DPPC, incorporated with MF, DMF and MF-ol shows significant interaction of these compounds with the alkyl chain of the hydrophobic core. MF, DMF and MF-ol acquire parallel orientation in bilayers with the B-ring pointing towards hydrophobic core, while DMF-ol acquire mixed orientation. This may be ascribed to the presence of two methoxy and one hydroxyl group on the B-ring of DMF-ol which hinders its partitioning inside the hydrophobic core of lipid bilayer. Multi-lamellar vesicles (MLV) of DPPC incorporated with flavones, show maximum increase in Chemical Shift Anisotropy in ${ }^{31} \mathrm{P}$ spectrum of DMF. This is followed by MF. DSC.

Conclusion: NMR and binding studies indicate that DMF is partitioned deeply inside the hydrophobic core, while MF, MF-ol and DMF-ol are mostly located in the vicinity of $s n$-glycero region. Therefore, we conclude that DMF which penetrates deepest inside the hydrophobic core also shows the highest anti-proliferative activity against K562 and MCF-7 cancer cell lines. Its activity is also better than CHY.

Keywords: Flavones, Flavonols, DPPC, DSC, NMR, Chemical shifts, CSA

(C) 2018 The Authors. Published by Innovare Academic Sciences Pvt Ltd. This is an open access article under the CC BY license (http://creativecommons.org/licenses/by/4.0/) DOI: http://dx.doi.org/10.22159/ijcpr.2018v10i2.25872

\section{INTRODUCTION}

Flavonoids are produced ubiquitously in plants. Several of them can be found in the diet or in traditional herbal medicines. Dietary flavonoids and other polyphenols have shown great potential as cancer chemopreventive agents in cell culture studies [1-3]. However, their oral bioavailability is poor due to which, they are hardly used as chemotherapeutic drugs $[4,5]$. The extensive conjugative metabolism in the intestine and liver is responsible for their poor in vivo activity [6,7]. The challenge is to modify the flavonoid moiety so as to facilitate the in vivo activity and thereby, increase their bioavailability. It has been reported [8] that replacement of hydroxyl group in flavones with methoxy groups reduce the degree of metabolic removal whilst retaining antiproliferative potency. 0-methylation provides polyphenols with protection against hepatic metabolism [9]. Their permeability across cell membranes is also increased [10]. Methoxy flavonoids are found naturally in plants $[11,12]$. Due to their increased lifespan in our body, methoxy flavonoids are much more capable to induce potential health effects in vivo as compared to their un-methoxylated counterparts [13]. Methoxy substituted flavonols have shown better antiinflammatory and anticancer activity against human prostate cancer cell lines as compared to their polyphenolic counterpart $[14,15]$.

The anti-proliferative activity of the methoxy flavonoids have been explored recently [16-18]. However, there are very few studies on their membrane interaction. It is known that the great bioactivity of flavonoids is closely linked to their ability to interact with membranes [19]. It is therefore important to study their localization and type of interaction inside the membranes. We have synthesized some mono-and di-methoxy flavones and flavonols [4'methoxyflavone (MF), 3', 4'-dimethoxyflavone (DMF), 4'-methoxy- flavonol (MF-ol), and 3', 4'-dimethoxyflavonol (DMF-ol)] (fig. 1). Their interaction, localization and orientation inside the lipid bilayer of DPPC model membranes have been studied. The interaction behavior, of methoxy flavonoids have been compared with the standard unmethoxylated polyphenols, chrysin (CHY) and quercetin (QUE). The antioxidant and anti-proliferative activity of methoxy flavonoids has also been studied and compared with CHY and QUE. A correlation between the interaction of the flavonoids with the lipid bilayer and their anti-proliferative activity has been established.
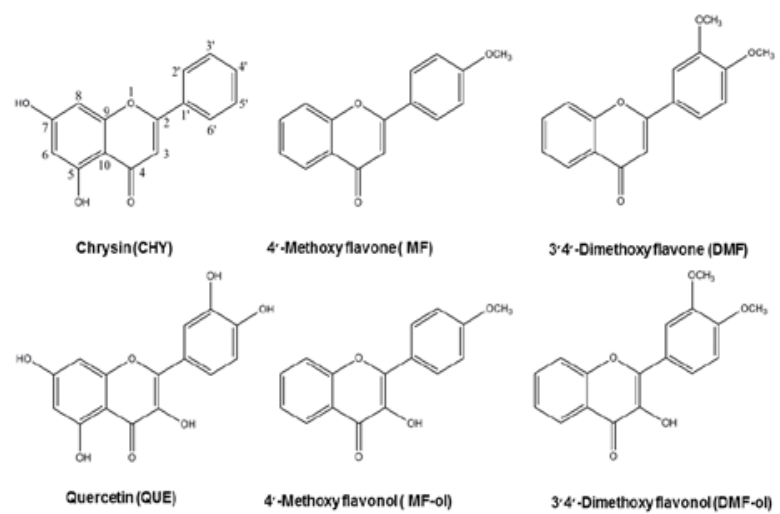

Fig. 1: Structure of Chrysin (CHY), 4'-Methoxyflavone (MF), 3', 4'Dimethoxyflavone (DMF), Quercetin (QUE), 4'-Methoxyflavonol (MF-ol), and 3', 4'-Dimethoxyflavonol (DMF-ol) 


\section{MATERIALS AND METHODS}

\section{Materials}

Chrysin, quercetin, 1, 2-dipalmitoyl-sn-glycero-3-phosphocholine (DPPC), 2, 2-diphenyl-1-picrylhydrozyl (DPPH), benzoic acid, phosphorous pentachloride $\left(\mathrm{PCl}_{5}\right)$ and 2-hydroxyacetophenone were purchased from Sigma chemicals Co. USA. The chemicals used for synthesis were purchased from SD Fine chemical, India and were of A. R. grade. The solvents used were of A. R grade.

\section{Synthesis}

The synthesis of compound 4'-methoxyflavone (MF) and 3', 4'dimethoxyflavone (DMF), was carried out according to the ClaisenSchmidt condensation reaction, using Lithium hydroxide ( $\mathrm{LiOH}$. $\mathrm{H}_{2} \mathrm{O}$ ) as a base catalyst. 4'-methoxyflavonol (MF-ol), and 3', 4'dimethoxyflavonol (DMF-ol) were synthesized from 2hydroxyacetophenone and 4-substituted benzaldehydes in two steps. (i) Claisen Schimdt reaction resulting in the formation of the chalcone. (ii) Algar-Finland-Oyamanda reaction resulting in the synthesis of the flavonols. A suspension of 4-methoxybenzaldehyde (for MF-ol) or 3, 4-dimethoxybenzaldehyde (for DMF-ol) and 2hydroxyacetophenone in ethanol was cooled and $\mathrm{KOH}$ solution was added dropwise. The reaction mixture was stirred at room temperature. $\mathrm{CH}_{2} \mathrm{Cl}_{2}$ was added and the organic layer was washed with $\mathrm{H}_{2} \mathrm{O}$, dried over sodium sulphate, and concentrated in vacou. The oily residue was dissolved in dioxane and ethanol and $\mathrm{NaOH}$ solution was added. To this $\mathrm{H}_{2} \mathrm{O}_{2}$ was added dropwise. The reaction mixture was stirred in an ice bath and subsequently at room temperature, resulting in a yellow suspension. After acidification with $2 \mathrm{M} \mathrm{HCl}$ the precipitate was filtered and dried. The crude product was purified by passing it through silica gel GF column using chloroform as mobile phase, to give pure products. The ${ }^{1} \mathrm{H}$ and
${ }^{13} \mathrm{C}$ chemical shifts of CHY, MF, DMF, QUE, MF-ol and DMF-ol in DMSO- $\mathrm{d}_{6}$ at $323 \mathrm{~K}$ are given in table 1.

\section{NMR and DSC experiments}

NMR experiments were recorded on a BRUKER AVANCE $500 \mathrm{MHz}$ NMR spectrometer. 2D-COSY and 2D-NOESY spectra were recorded using standard pulse sequences [20,21], with a mixing time of 400 ms.[31] $\mathrm{P}$ and ${ }^{13} \mathrm{C}$ experiments were carried out with a relaxation delay of $1 \mathrm{~s}$, using broadband ${ }^{1} \mathrm{H}$ decoupling. NMR software Topspin 2.0 was used for data processing. DSC measurements were performed on differential scanning calorimeter VP-DSC (Microcal, Northampton, MA, USA) using procedure used earlier [22]. Repeated scans for the same samples were generally super imposable. Data were analyzed with the software ORIGIN.

\section{Sample preparation}

Multi-lamellar vesicles (MLV's) from DPPC were prepared using standard procedure [23]. The desired quantity of DPPC and drug was dissolved in chloroform. The solvent was evaporated with a stream of nitrogen so as to deposit a lipid film on the walls of the container. The last traces of the solvent were removed under vacuum. MLV's sample thus prepared, was hydrated with the required amount of $\mathrm{D}_{2} \mathrm{O}$ at $\mathrm{pH} 7.2$, followed by incubation in water bath at $50{ }^{\circ} \mathrm{C}$ with repeated vortexing. The lipid concentration for NMR samples was maintained at $100 \mathrm{mmol}$. The concentrations of the flavones were varied from 10 to $50 \mathrm{mmol}$. For DSC experiments, samples were prepared by mixing the lipid and drug solutions to obtain drug/lipid ratios ranging from 1:20 to $1: 2$ by maintaining the lipid concentration to $50 \mathrm{mmol}$. Unilamellar vesicles (ULV's) for NMR experiments were prepared by sonicating the lipid dispersions, using a Branson Sonicator- 450 at $50 \%$ duty cycles till optical clarity was obtained.

Table 1: ${ }^{1} \mathrm{H}$ and ${ }^{13} \mathrm{C}$ NMR chemical shifts (ppm) for flavonoids in DMSO- $\mathrm{d}_{6}$ at $323 \mathrm{~K}$

\begin{tabular}{|c|c|c|c|c|c|c|c|c|c|c|c|c|c|}
\hline \multicolumn{2}{|l|}{${ }^{1} \mathrm{H}^{13} \mathrm{C}$} & \multicolumn{2}{|c|}{ CHY ${ }^{1} \mathrm{H}^{13} \mathrm{C}$} & \multicolumn{2}{|c|}{ MF $^{1} \mathrm{H}^{13} \mathrm{C}$} & \multicolumn{2}{|c|}{ DMF ${ }^{1} \mathrm{H}^{13} \mathrm{C}$} & \multicolumn{2}{|c|}{ QUE $^{1} \mathbf{H}^{13} \mathrm{C}$} & \multicolumn{2}{|c|}{ MF-ol ${ }^{1} \mathrm{H}^{13} \mathrm{C}$} & \multicolumn{2}{|c|}{ DMF-ol ${ }^{1} \mathrm{H}^{13} \mathrm{C}$} \\
\hline H3 & $\mathrm{C} 2$ & $6.9(\mathrm{~s})$ & 163.7 & $6.9(\mathrm{~s})$ & 162.8 & $7.0(\mathrm{~s})$ & 163.3 & $6.2(\mathrm{~s})$ & 147.4 & $8.1(d)$ & 146.1 & $8.1(\mathrm{~d})$ & 146.1 \\
\hline H5 & $\mathrm{C} 3$ & $6.2(\mathrm{~s})$ & 105.7 & $8.0(d)$ & 106.0 & $8.1(d)$ & 106.3 & $6.4(\mathrm{~s})$ & 136.2 & $7.5(\mathrm{t})$ & 133.9 & $7.5(\mathrm{t})$ & 138.9 \\
\hline H6 & $\mathrm{C} 4$ & $6.5(\mathrm{~s})$ & 182.3 & $7.8(\mathrm{t})$ & 177.5 & $7.5(\mathrm{t})$ & 177.5 & $7.7(\mathrm{~s})$ & 176.4 & $7.8(\mathrm{t})$ & 173.2 & $7.8(\mathrm{~b})$ & 177.3 \\
\hline $\mathrm{H} 7$ & $\mathrm{C} 5$ & $8.1(\mathrm{~d})$ & 162.0 & $7.5(\mathrm{t})$ & 123.9 & $7.8(\mathrm{t})$ & 124.0 & $6.9(\mathrm{~d})$ & 161.2 & $7.7(d)$ & 124.9 & $7.8(\mathrm{~b})$ & 124.0 \\
\hline H8 & C6 & $7.6(d)$ & 99.6 & $7.8(d)$ & 125.8 & $7.8(d)$ & 125.8 & $7.6(d)$ & 98.7 & $8.2(\mathrm{~s})$ & 125.1 & $7.8(\mathrm{~s})$ & 125.3 \\
\hline $\mathrm{H} 2^{\prime}$ & $\mathrm{C} 7$ & $7.6(\mathrm{~m})$ & 164.9 & $8.1(d)$ & 125.3 & $7.6(d)$ & 125.2 & $9.2(\mathrm{~s})$ & 164.4 & $7.1(\mathrm{~s})$ & 125.1 & $7.9(\mathrm{~d})$ & 125.0 \\
\hline H3' & $\mathrm{C} 8$ & $7.6(\mathrm{~m})$ & 94.6 & 7.1(d) & 118.9 & $7.2(\mathrm{~d})$ & 123.9 & $12.5(\mathrm{~s})$ & 93.8 & $7.2(\mathrm{~s})$ & 118.6 & $7.2(\mathrm{~d})$ & 119.0 \\
\hline $\mathrm{H} 4^{\prime}$ & C9 & $8.6(d)$ & 158.0 & 7.1(d) & 156.2 & 7.7 (d) & 156.2 & $10.7(\mathrm{~s})$ & 157.0 & $8.2(\mathrm{~s})$ & 154.9 & $3.9(\mathrm{~s})$ & 155.0 \\
\hline $\mathrm{H} 5^{\prime}$ & $\mathrm{C} 10$ & $12.7(\mathrm{~s})$ & 104.5 & 8.1(d) & 134.5 & 3.9 (d) & 134.5 & $9.2(\mathrm{~s})$ & 98.7 & $3.87(\mathrm{~s})$ & 138.5 & $9.5(\mathrm{~s})$ & 134.0 \\
\hline $\mathrm{H} 6^{\prime}$ & $\mathrm{C} 1^{\prime}$ & $10.7(\mathrm{~s})$ & 131.3 & $3.9(\mathrm{~s})$ & 131.5 & & 119.0 & $9.2(\mathrm{~s})$ & 122.5 & $9.26(\mathrm{~s})$ & 121.8 & & 121.9 \\
\hline $4^{\prime}-\mathrm{OCH}_{3}$ & $\mathrm{C} 2^{\prime}$ & & 126.9 & & 128.7 & & 110.3 & & 115.6 & & 129.8 & & 112.1 \\
\hline $3-\mathrm{OH}$ & $\mathrm{C} 3^{\prime}$ & & 129.6 & & 115.1 & & 152.6 & & 148.2 & & 114.5 & & 149.2 \\
\hline $5-\mathrm{OH}$ & $\mathrm{C} 4^{\prime}$ & & 132.4 & & 163.3 & & 149.7 & & 145.5 & & 160.9 & & 151.1 \\
\hline 7-OH & $\mathrm{C} 5^{\prime}$ & & 129.6 & & 115.1 & & 112.5 & & 116.1 & & 114.5 & & 112.4 \\
\hline $3^{\prime}-\mathrm{OH}$ & $\mathrm{C} 6^{\prime}$ & & 126.9 & & 128.7 & & 120.5 & & 115.7 & & 129.8 & & 122.1 \\
\hline $4^{\prime}-\mathrm{OH}$ & $\mathrm{CH}_{3}$ & & & & 56.1 & & $56.3,56.5$ & & & & 56.0 & & $56.2,56.3$ \\
\hline
\end{tabular}

\section{Determination of drug-MLV binding constants}

Drug-MLV binding constants were determined by centrifugation method [24]. MLV's were prepared by varying lipid concentration from $0.25 \mathrm{mg} / \mathrm{ml}$ to $2 \mathrm{mg} / \mathrm{ml}$ with fixed drug concentration of 100 $\mu \mathrm{M}$. This corresponds to a drug: lipid ratios in the range 1:2.5 to $1: 20$. The resulting solutions were incubated and subsequently transferred into ultracentrifuge tubes. Separation of liposomes from the aqueous phase was achieved by centrifugation. Optical density of $100 \mu \mathrm{M}$ solution of the pure drug was measured at a wavelength range of $220-400 \lambda$. The amount of drug bound to liposomes was determined from the difference in optical density measured for the pure drug and that of the supernatant. The drug-liposome apparent binding constant $(\mathrm{k})$ has been analyzed using the double reciprocal plot of fraction bound versus lipid concentration which yields a straight line with a slope $1 / \mathrm{k}$.

\section{Determination of antioxidant activity by DPPH assay}

Varying concentrations of each drug $(0-200 \mu \mathrm{g} / \mathrm{ml}, 0.5 \mathrm{ml}$ in methanol) was mixed with methanolic solution of DPPH $(0.1 \mathrm{mmol}$,
$1.0 \mathrm{ml})$ and Tris-HCl buffer $(0.1 \mathrm{M}, \mathrm{pH} 5.5,1.0 \mathrm{ml})$ to make a total volume $2.5 \mathrm{ml}$. The absorbance of the sample was measured at 517 $\mathrm{nm}$, after $0.5 \mathrm{~h}$ of incubation [25]. The reaction solution without DPPH was used as a blank test. Measurements were performed in triplicate. Free radical scavenging activity of the drug was measured as the difference in absorbance between the test sample and the control (i.e. sample without drug). Concentration required for a $50 \%$ reduction $\left(\mathrm{IC}_{50}\right.$ in $\mu \mathrm{g} / \mathrm{ml}$ ) of DPPH radical solution was determined graphically.

\section{Determination of anti-proliferative activity}

Anti-proliferative activity was evaluated by the Sulforhodamine B assay method [26]. Two different cell lines, K562 and MCF-7 were used. Cell lines were grown in RPMI 1640 medium containing 10\% fetal bovine serum and $2 \mathrm{mmol}$ of L-glutamine. The test compounds were dissolved in dimethyl sulfoxide and diluted suitably before adding them to the culture medium. After incubation at standard conditions for $48 \mathrm{~h}$, percent growth inhibition of cells has been calculated. 


\section{RESULTS AND DISCUSSION}

\section{Binding studies}

Fig. 2 shows a plot of fraction of drugs bound to MLV's with increasing concentration of lipid. In all cases, an increase in binding affinity is observed, with increasing concentration of lipid. $75-78 \%$ binding is shown by QUE, DMF and MF, CHY shows nearly $70 \%$ binding, while MF-ol and DMF-ol show only $62-65 \%$ binding at the highest concentration. Double inverse plot of fraction of drug bound vs inverse of lipid concentration (inset fig. 2) has been used to calculate the binding constants. The results indicate, that these molecules bind to the MLV's with a variable degree of affinity and follow the order QUE $>$ DMF $>$ MF $>$ CHY $>$ MF-ol $>$ DMF-ol. The apparent binding constants measured are: QUE: $1073 \mathrm{M}^{-1}$, DMF: $690 \mathrm{M}^{-1}$, MF: $559 \mathrm{M}^{-1}$, CHY: $472 \mathrm{M}^{-}$ ${ }^{1}$, MF-ol: $390 \mathrm{M}^{-1}$ and DMF-ol: $293 \mathrm{M}^{-1}$. It is noted that QUE, which has two hydroxyl groups on the B-ring, shows maximum binding. MF and DMF, which have one and two methoxy groups respectively on the Bring show considerable binding. MF-ol and DMF-ol show least binding. It appears that the penetration of the B-ring inside the lipid bilayer is responsible for the binding of flavonoids. The higher binding of flavones (MF and DMF) compared to that of flavonols (MF-ol and DMF-ol), may be due to the absence of- $\mathrm{OH}$ group on the B-ring. Possibly, presence of- $\mathrm{OH}$ group in addition to methoxy group on the B-ring, hinders its penetration inside the hydrophobic core. The methoxy groups present in B-ring of flavones may facilitate penetration. The binding results therefore suggest that the number and type of groups present on the B-ring of flavonoids play a significant role in their penetration inside the DPPC lipid bilayer.

\section{DSC studies}

DSC is a sensitive technique for studying the effect of drugs on the packing order of the lipid bilayers. Thermotropic aspect of drug-lipid interactions can be studied by examining changes in the melting point and the shape of the DSC thermograms [27]. Fig. 3 shows DSC thermograms of lipid bilayers incorporated with increasing concentrations of CHY, MF, DMF, QUE, MF-ol and DMF-ol. In each case, the lower most graphs (a) represent the thermogram for lipid bilayers alone. Here, the pre-transition temperature $\left(\mathrm{T}_{\mathrm{p}}\right)$ at $34.1^{\circ} \mathrm{C}$ indicates the mobility of the choline part of the polar head of DPPC. Mobility of the alkyl chain is reflected in the main transition $\left(\mathrm{T}_{\mathrm{m}}\right)$ at $41.9^{\circ} \mathrm{C}$. In the case of flavonols QUE and MF-ol the pre-transition peak is abolished. Abolition of such a peak, indicates a strong interaction of these molecules, with the polar head-group region of the lipid bilayer. However, pretransition peak is present in thermogram of MF, DMF and DMF-ol. The presence of such a peak indicates the interaction of these molecules with head group region of lipid bilayer [28]. In the case of MF and DMF the value of pretransition peak decreases with increase in concentration of drug, but in CHY and DMF-ol it initially decreases and at concentrations higher than 1:10 (drug: lipid molar ratio) it increases.

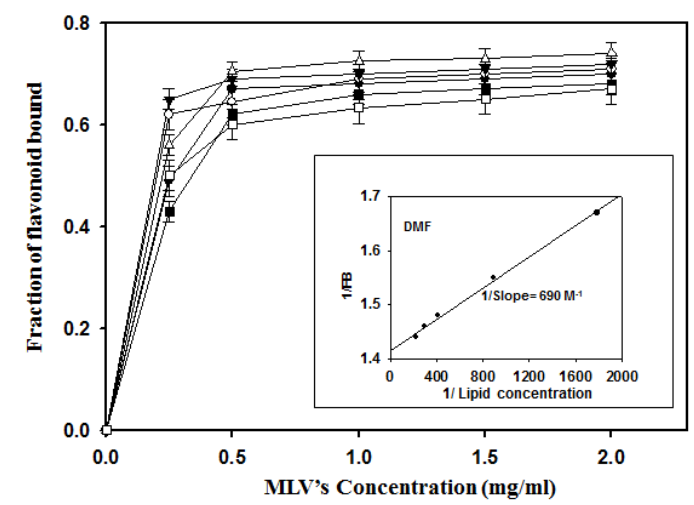

Fig. 2: Binding of $\operatorname{CHY}(O), \operatorname{MF}(O, \operatorname{DMF}(\nabla), \operatorname{QUE}(\Delta, \operatorname{MFOl}(\square)$ and DMF-ol ( $\square$ ), with DPPCMLV's. The fraction of drug bound (FB) has been determined by the centrifugation method, as described in the text. The inset fig. shows the double reciprocal plot for DMF

A decrease in the main transition temperature on addition of the compounds indicates an interaction of the molecules with the hydrophobic core of the lipid bilayer. The maximum decrease in $\mathrm{T}_{\mathrm{m}}$ value is observed for DMF (at drug/lipid molar ratio of 1:2) i.e. 3.7 ${ }^{\circ} \mathrm{C}$ as compared to pure DPPC bilayer (table 2), indicating a strong interaction with the hydrophobic core which enhances the fluidity of the lipid bilayers.

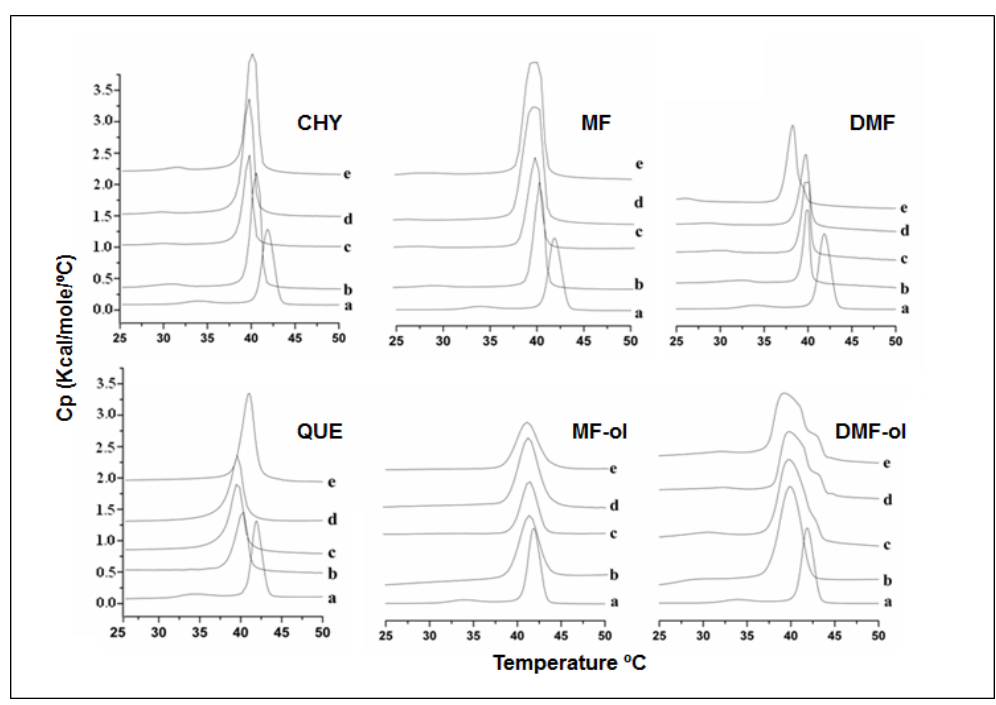

Fig. 3: DSC heating curves of hydrated MLV's of DPPC (50 mmol) containing drugs, at drug/lipid molar fractions; a=0.0; b=0.05; c=0.1; $d=0.2 ; \mathrm{e}=0.5$

Table 2: Pretransition ( $\left.T_{p}\right)$ and main transition $\left(T_{m}\right)$ temperatures of DPPC (50 mmol) with varying drug/lipid molar ratios

\begin{tabular}{|c|c|c|c|c|c|c|c|c|c|c|c|c|}
\hline \multirow{2}{*}{$\begin{array}{l}\text { Drug/DPPC } \\
\text { Pure DPPC }\end{array}$} & \multicolumn{2}{|c|}{$\mathrm{CHY} \mathrm{T}_{\mathrm{p}} \mathrm{T}_{\mathrm{m}}$} & \multicolumn{2}{|c|}{$\mathbf{M F}_{\mathbf{p}} \mathbf{T}_{\mathrm{m}}$} & \multicolumn{2}{|c|}{ DMF T $_{p} T_{m}$} & \multicolumn{2}{|c|}{ QUE T $_{p} T_{m}$} & \multicolumn{2}{|c|}{ MF-ol $T_{p} T_{m}$} & \multicolumn{2}{|c|}{ DMF-ol $T_{p} T_{m} T_{m 1}$} \\
\hline & 34.1 & 41.9 & 34.1 & 41.9 & 34.1 & 41.9 & 34.1 & 41.9 & 34.1 & 41.9 & 34.1 & 41.9-- \\
\hline $1: 20$ & 31.0 & 40.6 & 29.3 & 40.3 & 32.5 & 40.0 & -- & 40.2 & -- & 41.5 & 29.6 & 39.9-- \\
\hline $1: 10$ & -- & 39.8 & 28.3 & 39.8 & 30.6 & 39.9 & -- & 39.7 & -- & 41.4 & 30.5 & 39.841 .7 \\
\hline $1: 5$ & 30.5 & 39.9 & 28.0 & 39.7 & 28.9 & 39.7 & -- & 39.6 & -- & 41.2 & 32.1 & 39.741 .9 \\
\hline $1: 2$ & 31.6 & 40.1 & -- & 39.7 & 26.3 & 38.2 & -- & 39.7 & -- & 41.1 & 32.5 & 39.242 .8 \\
\hline
\end{tabular}


This is followed by MF with maximum decrease of $2.2{ }^{\circ} \mathrm{C}$. A broadening of $\mathrm{T}_{\mathrm{m}}$ peak in the case of MF indicates a decrease in cooperativity of the alkyl chain again indicating its localization, in the hydrophobic core.

In case of CHY and QUE the $T_{m}$ value decreases up to a concentration of 1:5 (CHY/QUE: lipid) molar ratio after which it increases. This can be explained by the tendency of QUE and CHY to form aggregates when in excess of their solubility in lipid bilayers [29].

The thermogram of MF-ol shows a decrease in $\mathrm{T}_{\mathrm{m}}$ with increase in concentration however, the decrease in value is less than that of MF and DMF. This indicates interaction with the hydrophobic core of lipid bilayer. DMF-ol shows a broadening of the main transition peak and appearance of an additional shoulder peak. This new transition peak is observed at a temperature higher than the main transition peak. As concentration of DMF-ol is increased from 1:10 (drug: lipid) to 1:2 (drug: lipid) molar ratio the new peak becomes more prominent. This behavior indicates domain formation and phase separation at higher concentrations of DMF-ol. High concentration of DMF-ol probably induces a heterogeneous system [30]. This means that a homogeneous distribution of the compound within the DPPC bilayers did not occur but rich and poor compound regions are present. It also explains the increase in value of pretransition peak of DMF-ol with increase in drug concentration.

The above DSC results indicate maximum interaction of MF and DMF with the hydrophobic core. DMF-ol shows interaction with the head group as well as with the alkyl chain of the lipid bilayer at lower drug concentration, but finally forms a heterogeneous system at higher concentrations.

\section{NMR experiments}

${ }^{1} \mathrm{H}$ and ${ }^{13} \mathrm{C}$ NMR spectra of CHY, MF, DMF, QUE, MF-ol and DMF-ol in DMSO- $\mathrm{d}_{6}$ have been assigned using 2D COSY spectrum, literature data and splitting patterns (table 1). Fig. 4 shows aromatic region of ${ }^{1} \mathrm{H}$ NMR spectra of drugs alone (spectra a, b, c, d, e, and f) and of drugs incorporated with ULVs of DPPC (spectra $a^{\prime}, b^{\prime}, c^{\prime}, d^{\prime}, e^{\prime}$ and $\left.\mathrm{f}^{\prime}\right)$. It is observed that in all cases, the drug signals arising from aromatic protons become broad on incorporation into lipid bilayers. There are significant amount of chemical shift changes both downfield and up-field in all cases. This is because the molecule is likely to interact by spanning from head to tail region of lipid bilayers involving interaction with both polar as well as hydrophobic chain region. Aromatic protons of MF, DMF and MF-ol show both up-field and downfield shifts. $8 \mathrm{H}$ and $6 \mathrm{H}$ protons of $\mathrm{MF}$ show significant up-field and downfield shifts respectively, indicating strong interactions at these positions with lipid bilayer. DMF and MF-ol when incorporated in DPPC bilayers show significant downfield chemical shift of $5 \mathrm{H}$ proton indicating strong interactions at this position. The aromatic region of DMF-ol show distinct behavior than that of MF, DMF and MF-ol on interaction with lipid molecules. In ${ }^{1} \mathrm{H}$ NMR of ULV of DPPC incorporated with DMF-ol, the peaks of the aromatic region are broadened significantly and additional peaks are observed. This may be due to formation of two different domains as we have discussed in our DSC results. Based on the peak intensity as well as their ${ }^{1} \mathrm{H}-{ }^{1} \mathrm{H}$ NOESY spectrum we have characterized the peaks of DMF-ol. Here again, we observe a significant downfield shift of $5 \mathrm{H}$ proton indicating strong interaction at this position. CHY and QUE show only downfield shift of the protons as these molecules are likely to bind at the lipid water interface due to presence of hydroxyl groups on the ring A. Presence of hydroxyl group on ring-A mostly shifts the location of the ring to lipid water interface [31,32].

A comparison of the ${ }^{1} \mathrm{H}$ NMR spectra of pure DPPC and ULV's of DPPC incorporated with drugs indicates shift of DPPC protons on binding with flavonoids under study (fig. 5). These shifts (so called induced chemical shifts), can be used for predicting the localization of the compounds in the lipid bilayer. Upon binding with CHY and QUE there is significant shift in all the regions of lipid but the largest shift is in the $s n$-glycero region of the lipid water interface. This indicates their localization in this region. Uniform up-field shift is induced in the DPPC protons on incorporation with MF, DMF and MF-ol (fig. 5). However, interaction of MF and DMF is in the alkyl chain region as well as with the sn-glycero region whereas, that of MF-ol is only in the sn-glycero region. In case of MF-ol and DMF-ol the interaction observed with alkyl and sn-glycero region is comparatively less.

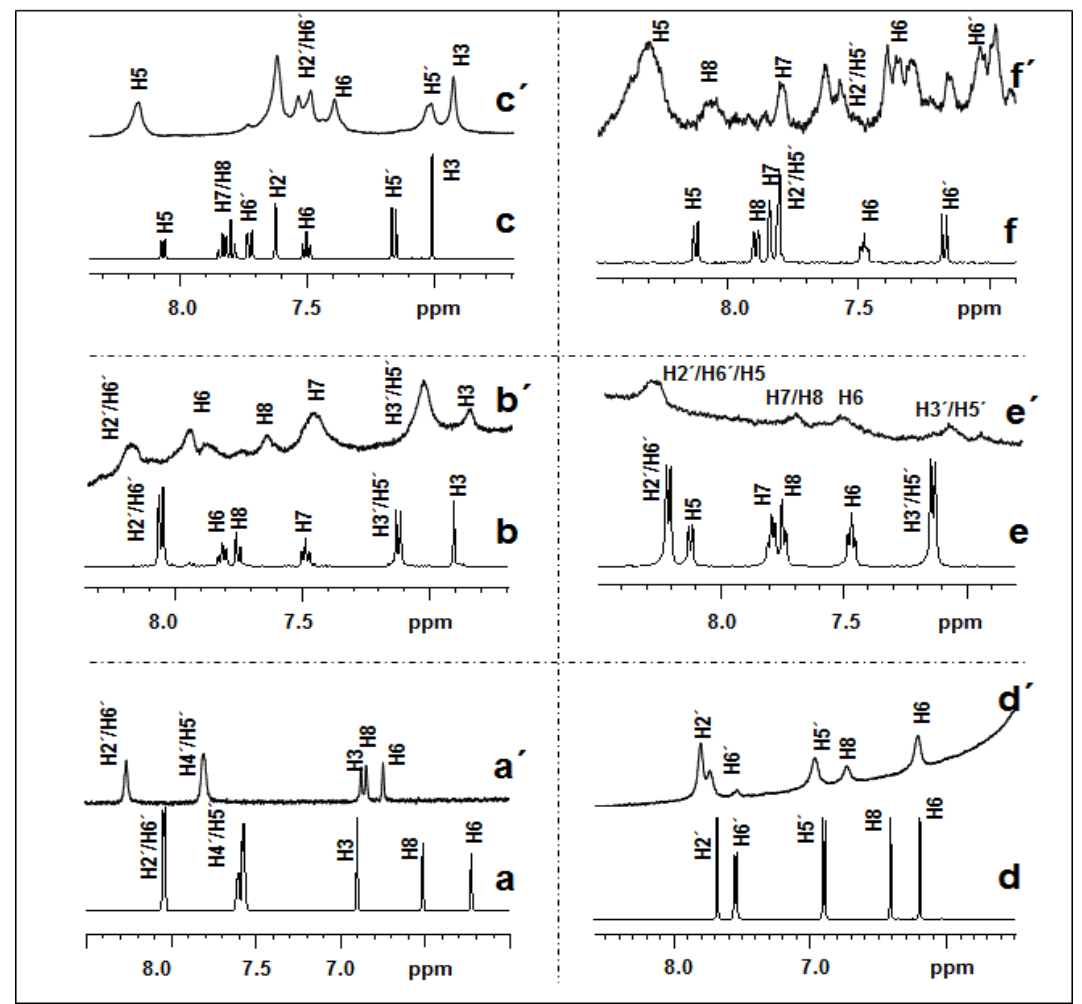

Fig. 4: $500.13 \mathrm{MHz}^{1} \mathrm{H}$ NMR spectra of aromatic region of pure CHY (a), MF (b), DMF (c), QUE (d), MF-ol (e) and DMF-ol (f) and that of ULV's of DPPC incorporated with CHY ( $\left.a^{\prime}\right)$, MF ( $\left(b^{\prime}\right)$, DMF ( $\left.c^{\prime}\right), Q U E$ ( $\left.d^{\prime}\right)$, MF-ol ( $e^{\prime}$ ) and DMF-ol ( $\left.f^{\prime}\right)$ in 1:5 drug: lipid molar ratio at $323 \mathrm{~K}$ in $\mathrm{D}_{2} \mathrm{O}$ 


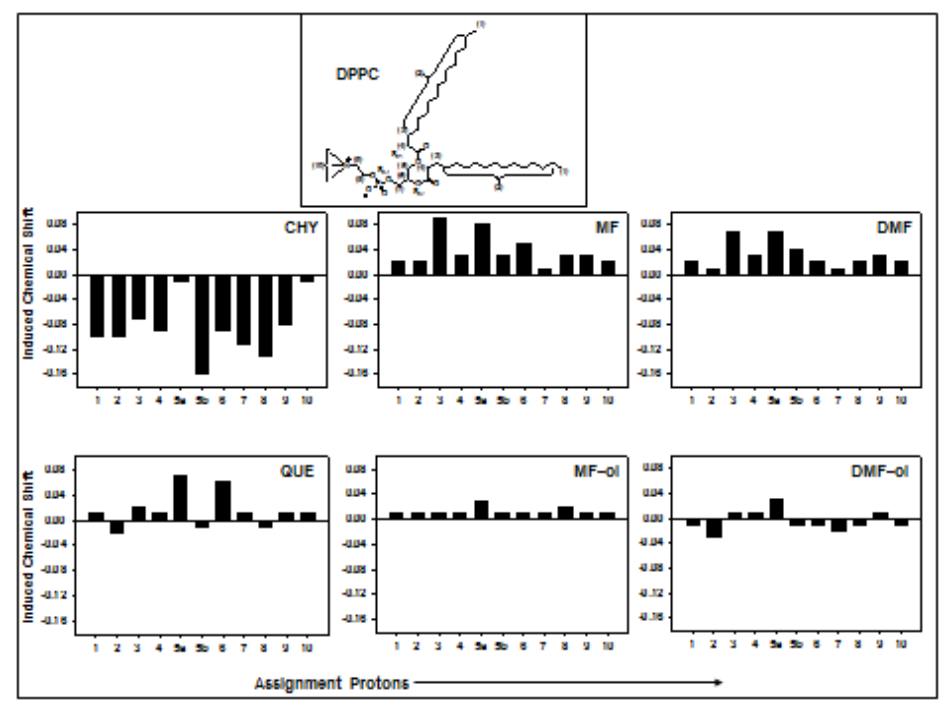

Fig. 5: Ring current induced chemical shift changes in the signals of ULV's of DPPC incorporated with drugs in 1:5 drug/lipid molar ratio at $323 \mathrm{~K}$ in the presence of CHY, MF, DMF, QUE, MF-ol and DMF-ol. Chemical shifts are either up-field (+) or downfield (-), depending on the orientation of the drug molecule with respect to the bilayer normal

The induced chemical shifts of the lipid protons also indicate the orientation of the drug molecules with respect to the lipid bilayer normal [33]. Drug molecules impart ring current effects on the lipid protons, due to which the lipid resonances are known to shift upfield or downfield. Upon interaction with ring edge the resonances are shifted downfield and on interaction with ring centre it is shifted up-field [34]. Thus, a uniform downfield shift indicates the ring plane to be perpendicular whereas a uniform up-field shift indicates a parallel orientation of the ring plane with reference to the bilayer normal. A mixed orientation of the rings is indicated by the absence of a uniform up-field or downfield shift. A perpendicular orientation is seen in case of CHY with respect to the lipid bilayer normal. MF, DMF and MF-ol show a parallel orientation. QUE and DMF-ol show mixed orientation with respect to bilayer normal.

To probe deeper into the nature of interaction, ${ }^{1} \mathrm{H}^{-1} \mathrm{H}$ NOESY spectra of ULV's of DPPC and of those incorporated with drugs have been used. In the spectra of DMF incorporated with ULV's, intermolecular NOE of the methoxy protons with the- $\left(\mathrm{CH}_{2}\right)_{\mathrm{n}}$ protons of lipid bilayer is observed (fig. not shown). This indicates interaction of the methoxy group protons with the hydrophobic core of DPPC bilayer. Though the NOESY spectrum of DMF-ol was noisy, but we were able to locate the intermolecular NOEs of $\mathrm{H}^{\prime}, \mathrm{H}^{\prime}$ with the head region- $\mathrm{N}\left(\mathrm{CH}_{3}\right)_{3}$ protons. This indicates that DMF-ol molecule largely resides in the polar head region with some penetration in the hydrophobic core.

In the ${ }^{13} \mathrm{C}$ NMR of the DPPC ULV's and that of ULV's incorporated with CHY, MF, DMF, QUE, MF-ol and DMF-ol, all the signals arising from drugs are broadened compared to their pure form (fig. 6). The broadening of the signals arises due to an exchange at an intermediate NMR time scale between the bound and free form of these molecules [35]. On the other hand, signals arising from lipid bilayers mostly do not broaden. However, broadening of lipid peaks are observed in the spectrum of ULV vesicles incorporated with MF, DMF and DMF-ol in the region belonging to- $\left(\mathrm{CH}_{2}\right)_{n},-\left(\mathrm{CH}_{2}\right)_{\beta}$ and $-\left(\mathrm{CH}_{2}\right)_{\alpha}$ groups of the alkyl chain of the lipid showing its interaction with the drug molecule (fig. 6). In ULV vesicles incorporated with other compounds, these peaks remain sharp. This supports our observation that MF, DMF and DMF-ol interact with the alkyl chain of the hydrophobic core. In the case of DMF, QUE and DMF-ol the C5 carbon of the sn-glycero region (indicated by * in fig. 6) shows significant up-field shift in its position, thereby indicating interaction of the drug molecule at this position. The ${ }^{13} \mathrm{C}$ spectrum of DMF-ol shows several peaks in the aromatic region inspite of broadening, indicating mobility of these atoms. In other words, the aromatic protons present on these carbon atoms of DMF-ol is not involved in interaction with the lipid protons and thus these carbon atoms give rise to sharp carbon peaks in their ${ }^{13} \mathrm{C}$ NMR spectrum.

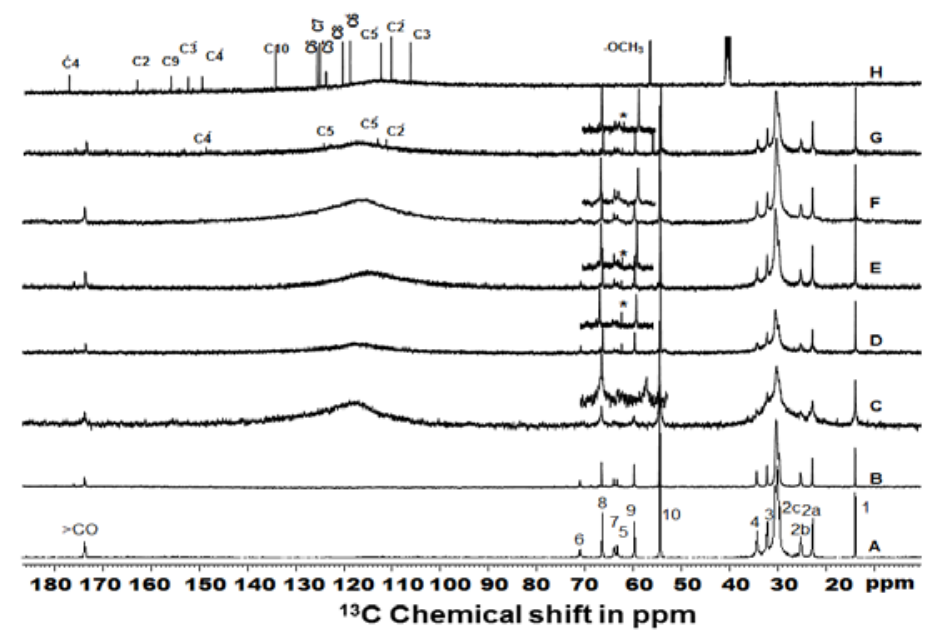

Fig. 6: 125.7 MHz ${ }^{13} \mathrm{C}$ NMR spectra of DPPC ULV's (A) and ULV's of DPPC incorporated with CHY (B), MF (C), DMF (D), QUE (E), MF-ol (F) and DMF-ol (G) in 1:5 drug/lipid molar ratio at $323 \mathrm{~K}$ in $\mathrm{D}_{2} \mathrm{O}$. The ${ }^{13} \mathrm{C}$ NMR spectrum of pure DMF-ol (H), in DMSO-d 6 is also shown. Peaks for carbon atoms 5 and 7 have been vertically expanded to show upfield shift as indicated by* 
31P NMR is sensitive to local motions and the orientation of the phosphate group in the membrane. It has been used for monitoring structural changes and detecting polymorphism in model membranes [36]. Effect of CHY, MF, DMF, QUE, MF-ol and DMF-ol on [31]P line shape has been monitored at varying temperature and concentrations. It may be noted here that these molecules do not alter the characteristic line shapes exhibiting bilayer features of the MLV's of lipid at all concentrations (Supplementary Fig.1). Lipid bilayers give a characteristic broad spectrum with a high field peak and a low field shoulder. Chemical shift anisotropy (CSA) can be measured from the low and high field shoulders of the spectrum $\left(\sigma \mid\right.$ and $\sigma_{\perp}$ components). The CSA of the phosphate group is used to determine molecular motions near the bilayer head groups [37].

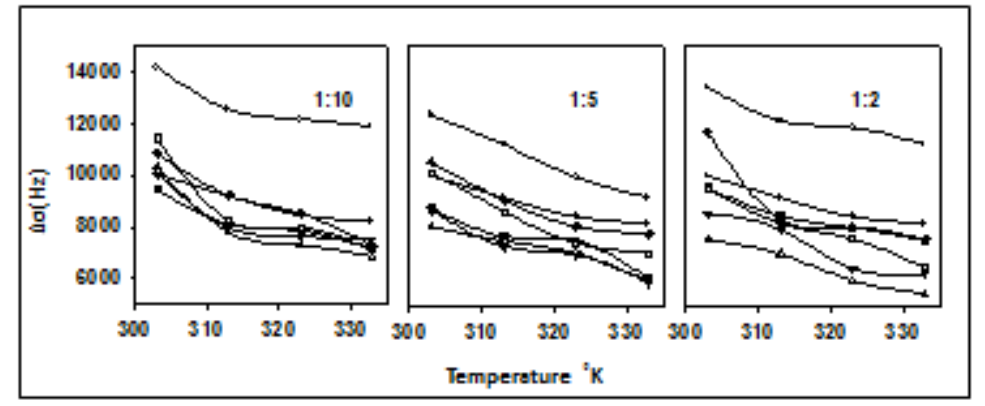

Fig. 7: Change in CSA (Hz) represented by $\Delta \sigma=\sigma \perp-\sigma \perp$ with temperature at 1:10, 1:5 and 1:2 drug: lipid molar ratios. DPPC MLVs (O), CHY $(\mathrm{O}), \operatorname{MF}(\nabla), \operatorname{DMF}(\Delta, \mathrm{QUE}(\boldsymbol{\nabla}), \mathrm{MFol}(\square)$ and DMF $(\diamond)$

In case of CHY, QUE and DMF-ol the CSA values decreases upto 1:5 (drug: lipid) molar ratios and then increase at 1:2 (drug: lipid) molar ratio. This may be due to formation of aggregates at higher concentration of drug [29]. This fact is supported by our DSC results.

Thus, based on binding, NMR and DSC studies we can assume that the A-ring of flavonols and flavones are probably located at the lipid/water interface whereas B-ring penetrates into the hydrophobic core of lipid bilayer. MF and DMF is located deep in the hydrophobic core followed by MF-ol. The binding as well as interaction of DMF-ol is hindered by its formation of a heterogeneous system at higher concentrations on incorporation inside the lipid vesicles. Further, DMF-ol shows a mixed orientation with respect to lipid bilayer normal like QUE.

\begin{abstract}
Anti-oxidant and anti-proliferative activity
DPPH assay has been used to estimate the radical scavenging and antioxidant activity of the flavonols [39]. The radical scavenging activity is known to depend on the number and the position of the phenolic-OH groups [40]. CHY, MF, DMF, QUE, MF-ol and DMF-ol possess $\mathrm{OH}$ groups and show highest antioxidant activity. The antioxidant property of these molecules follows the order: QUE $>$ CHY $>$ MF-ol $>$ DMF-ol $>$ MF $>$ DMF (table 3 ), depending on the presence of hydroxyl group. The anti-proliferative activity of the flavones is shown against K562 and MCF7 cell lines in table 3.
\end{abstract}

Table 3: In-vitro antioxidant and anti-proliferative activity of flavonoids

\begin{tabular}{llll}
\hline Flavonoids & DPPH radical scavenging activity $\left.\mathbf{I C}_{\mathbf{5 0}} \mathbf{(} \mathbf{\mu g} / \mathbf{m l}\right)^{\mathbf{a}}$ & Percent growth compared to control against different cell lines $^{\mathbf{b}}$ \\
\cline { 3 - 4 } & & $\mathbf{K 5 6 2}$ & $\mathbf{\mathbf { M C F } ^ { - 7 }}$ \\
\hline CHY & 20.40 & 25.9 & 38.3 \\
MF & 46.37 & 25.4 & 66.8 \\
DMF & 50.20 & 13.9 & 14.4 \\
QUE & 9.14 & 12.4 & 8.3 \\
MF-ol & 34.20 & 32.6 & 24.9 \\
DMF-ol & 42.10 & 51.2 & 42.9 \\
ADR & - & -53.4 & 14.9 \\
\hline
\end{tabular}

aThe molar drug concentrations required to cause $50 \%$ inhibition $\left(\mathrm{IC}_{50}\right)$ were determined from, dose-response curves. Results represent means $\pm \mathrm{SE}$ of at least three different experiments, ${ }^{\mathrm{b}}$ concentration of drugs in each case is $10^{-4} \mu \mathrm{g} / \mathrm{ml}$.

Though QUE interacts significantly only with the sn-glycero region of DPPC, it shows highest anti-proliferative activity against both the cell lines. DMF shows better activity as compared to rest of the compounds. MF shows better activity than MF-ol and DMF-ol against K562. However higher activity is shown by MF-ol as compared to MF against MCF-7 cell line. Therefore, after studying the activity of all the synthesized compounds we can say that, DMF which shows maximum interaction with the hydrophobic core of lipid bilayer also shows better activity as compared to other synthesized compounds, including the standard compound chrysin.

\section{CONCLUSION}

The objective of the present study was to establish the effect of substitution at $4^{\prime}$-as well as $3^{\prime}$ and $4^{\prime}$-position of the flavones and flavonols on their pharmacological activity. It is proved that localization and interaction of these molecules plays an important role in their activity. DMF has a strong binding with the lipid hydrophobic core and imparts maximum activity. MF and MF-ol which binds mostly at the lipid/water interface are less active. DMFol forms a heterogeneous system on incorporation inside lipid bilayers, which may hinder better interaction with lipid bilayers. It is less active against cancer cell lines K562 and MCF-7. We can therefore, conclude that substitution of a methoxyl group at 4 and/or 3'-position on the B-ring of the flavone moiety facilitates its partitioning into the hydrophobic core to some extent. This may enhance its anti-proliferative activity against selective cancer cell lines as well. This is also supported by literature reports by Walle et al. [41], where they have shown that 5, 7-dimethoxy flavone shows better anti-proliferative activity as well as in-vivo bioavailability as compared to their unmethoxylated counterpart. Substitution of a methoxyl group at $4^{\prime}$-position on the B-ring of flavonols, also enhances its activity as well as partitioning into the hydrophobic core of lipid bilayer. Substitution of two methoxyl groups on the B- 
ring of flavonol may create steric hinderance in its partitioning inside the lipid bilayer. It is concluded that the substitution of methoxy groups in flavones and flavonols increases its potential as a bioactive compound. The position and number of methoxy group, also play an important role. However, more flavonoid molecules need to be evaluated and other cancer cell lines should be used in order to draw definite conclusions about these structural requirements in regard to methoxylation and antiproliferative activity. These findings can be useful to direct synthesis of new flavonoid-methoxy analogs towards prevention and/or treatment of cancer.

\section{ACKNOWLEDGEMENT}

Ragini Sinha and Girjesh Govil thank the Indian National Science Academy, and Urmila Joshi, Heena R. Bhojwani and Akshada Joshi, thank Department of Biotechnology, for funding the above project. The help provided by National Facility for High Field NMR located at TIFR are gratefully acknowledged.

\section{AUTHORS CONTRIBUTIONS}

All the author have contributed equally

\section{CONFLICT OF INTERESTS}

\section{Declared none}

\section{REFERENCES}

1. Kandaswami C, Perkins E, Soloniuk DS, Drzewieki G, Middleton E. Antiproliferative effects of citrus flavonoids on a human squamous cell carcinoma in vitro. Cancer Lett 1991;56:147-52.

2. Middleton EJ, Kandaswami C, Theoharides TC. The effect of plant flavonoids on mammalian cells: implications for inflammation, heart disease and cancer. Pharmacol Rev 2000;52:673-571.

3. Pervaiz S. Resveratrol: from grapevines to mammalian biology. J FASEB 2003;17:1975-85.

4. Goldberg DM, Yan J, Soleas GJ. Absorption of three wine related polyphenols in three different matrices by healthy subjects. Clin Biochem 2003;36:79-87.

5. Kanoo M, Takayanagi T, Harada K, Sawada S, Ishikawa F. Bioavailability of isoflavones after ingestion of soy beverages in healthy adults. J Nutr 2006;136:2291-6.

6. Manach C, Scabert A, Morand C, Remesy C, Jiminez L. Polyphenols: food sources and bioavailability. Am J Clin Nutr 2004;79:727-47.

7. Scalbert A, Williamson G. Dietary intake and bioavailability of polyphenols. J Nutr 2000;130:2073-85.

8. Cai H, Sali S, Schmid R, Britton RG, Brown K, Steward WP, et al. Flavones as colorectal cancer chemopreventive agents-phenol0-methylation enhances efficiency. Can Prev Res 2009;2:743-50.

9. Wen X, Walle T. Methylated flavonoids have greatly improved intestinal absorption and metabolic stability. Drug Metab Disposition 2006;34:1786-92.

10. Artursson P, Karlsson J. Correlation between oral drug absorption in humans and apparent drug permeability coefficients in human intestinal epithelial (caco-2) cells. Biochem Biophys Res Comm 1991;175:880-5.

11. Mizuno $M$, Iinuma $M$, Ohara $M$, Tanaka $T$, Iwamasa $M$. Chemotaxonomy of the genus citrus based on polymethoxy flavones. Chem Pharm Bull 1991;39:945-9.

12. Jaipetch T, Reutrakul V, Tuntiwachwuttikul P, Santisuk T. Flavonoids in the black rhizomes of boesenbergia pandurate. Phytochem 1983;22:625-6.

13. Britton RG, Horner-Glister E, Pomenya OA, Smith EE, Denton R, Jenkins PR, et al. Synthesis and biological evaluation of novel flavonols as potential anti-prostrate cancer agents. Eur J Med Chem 2012;54:952-8.

14. During A, Larondelle Y. The O-methylation of chrysin markedly improves its intestinal anti-inflammatory properties: structure-activity relationships of flavones. Biochem Pharmcol 2013;86:1739-46.

15. Saad S, Howells LM, Britton RG, Steward WP, Gescher A, Brown K. Sale S, 3', 4', 5'-trimethoxyflavonol (TMFol), a novel putative prostrate cancer chemopreventive agent; in-vitro and in-vivo preclinical activity. Can Prev Res 2010;3:A104.

16. Pouget C, Lauthier F, Simon A, Fagnere C, Basly JP, Delage C. Flavonoids structural requirements for antiproliferative activity on breast cancer cells. Bioorg Med Chem Lett 2001;11:3095-7.

17. Manthey JA, Guthrie N. Antiproliferative activities of citrus flavonoids against six human cancer cell lines. J Agric Food Chem 2002;50:5837-43.

18. Howells LM, Britton RG, Mazzoletti M, Greaves P, Broggini M, Brown K, Steward WP, Gescher AJ, Sale S. Preclinical colorectal cancer chemopreventive efficacy and p 53-modulating activity of 3', 4', 5'-trimethoxyflavonol, a quercetin analogue, Can Prev Res 2010;3:929-39.

19. Tsuchiya H. Structure-dependent membrane interaction of flavonoids associated with their bioactivity. Food Chem 2010;120:1089-96.

20. Aue WP, Bartholdi E, Ernst R. Two dimensional spectroscopy. Application to nuclear magnetic resonance. J Chem Phys 1976;64:2229-46.

21. Anet FAL, Bourn AJR. Nuclear magnetic resonance spectral assignments from nuclear overhauser effects. J Am Chem Soc 1965;87:5250-1.

22. Sinha R, Gadhwal MK, Joshi UJ, Srivastava S, Govil G. Interaction of quercetin with DPPC model membrane: Molecular dynamic simulation, DSC and multinuclear NMR studies. J Indian Chem Soc 2011;88:1203.

23. Kornberg RD, McConnel HM. Inside-outside transitions of phospholipids in vesicle membranes. Biochemistry 1971;10:1111-20.

24. Cheng HY, Randall CS. Carvedilol-liposome interaction: evidence for strong association with the hydrophobic region of the lipid bilayers. BBA-Biomembranes 1996;1284:20-8.

25. Molyneux $P$. The use of the stable free radical diphenylpicrylhydrazyl (DPPH) for estimating antioxidant activity. J Sci Technol 2004;2:211-9.

26. Vichai V, Kirtikara K. Sulforhodamine B colorimetric assay for cytotoxicity screening. Nat Protocols 2006;1:1112-6.

27. Lambros MP, Sheu E, Lin JS, Pereira HA. Interaction of a synthetic peptide based on the neutrophil-derived antimicrobial protein CAP37 with dipalmitoylphosphatidylcholine membranes. BBA-Biomembranes 1997;1329:285-90.

28. Sinha R, Gadhwal MK, Joshi A, Joshi UJ, Srivastava S, Govil G. Structure-dependent interaction of hydroxy flavones with DPPC model membrane. Eur J Med Chem 2014;80:285-94.

29. Sinha R, Gadhwal MK, Joshi A, Joshi UJ, Srivastava S, Govil G. Localization and interaction of hydroxy flavones with lipid bilayer model membranes: a study using DSC and multinuclear NMR. Eur J Med Chem 2014;80:285-94.

30. Gardikis K, Hatziantoniou S, Viras K, Demetzos C. Effect of a bioactive curcumin derivative on DPPC membrane: a DSC and raman spectroscopy study. Thermochim Acta 2006;447:1-4.

31. Bassolino-Klimas D, Alper HE, Stouch TR. Mechanism of solute diffusion through lipid bilayer membranes by molecular dynamics simulation. J Am Chem Soc 1995;117:4118-29.

32. Kachel K, Asuncion-Punzalan E, London E. Anchoring of tryptophan and tyrosine analogs at the hydrocarbon-polar boundary in model membrane vesicles. Biochemistry 1995;34:15475-9.

33. Kimura T, Cheng K, Rice KC, Gawrisch K. Location, structure, and dynamics of the synthetic cannabinoid ligand CP-55,940 in lipid bilayers. Biophys J 2009;96:4916-24.

34. Stamm H, Jaeckel H. Relative ring current effects based on a new model for aromatic-solvent-induced shift. J Am Chem Soc 1989;111:6544-50.

35. Levine YK, Partington P, Roberts GCK, Birdstall NMJ, Metcalfe JC. 13C nuclear magnetic relaxation times and models for chain molecules in lecithin vesicles. FEBS Lett 1972;23:203-7.

36. Srivastava S, Phadke RS, Govil G. Role of tryptophan in inducing polymorphic phase formation in lipid dispersions. Indian J Biochem Biophys 1988;25:283.

37. Cullis PR, Hope MJ, Tilcock CPS. Lipid polymorphism and the role of lipids in membranes. Chem Phys Lipids 1986;40:12744. 
38. Frenzel J, Arnold K, Nuhn P. Calorimetric, 13C NMR and 31P NMR studies on the interaction of some phenothiazine derivatives with dipalmitoyl phosphatidylcholine model membranes. Biochem Biophys Acta 1978;507:185-97.

39. Brand-Williams W, Cuvelier ME, Berset C. Use of a free radical method to evaluate antioxidant activity. Lebensm Wiss Technol 1995;28:25-30.
40. Van-Acker SABE, de Groot MJ. A quantum chemical explanation of the antioxidant activity of flavonoids. Chem Res Toxicol 1996;9:1305-12.

41. Walle T, Ta N, Kawamori T, Wen X, Tsuji PA, Walle UK. Cancer chemopreventive properties of orally bioavailable flavonoidsethylated versus unmethylated flavones. Biochem Pharmaco 2007;73:1288-96. 